\title{
Prevalência de pterígio e pinguécula em Populações quilombolas da Amazônia Brasileira
}

\author{
Prevalencia de pterigio y pinguécula en Poblaciones quilombolas de la Amazonia \\ Brasileña \\ Prevalence of pterygium and pinguécula in Quilombola populations of the Brazilian \\ Amazon
}

Paula Renata Caluff Tozzatti ${ }^{1}$, Jefison da Silva Lopes ${ }^{2 *}$, Sabrina Macambira Guerra da Rocha ${ }^{2}$, Iris Santos de Souza², Camila de Almeida Pereira², Natasha Cortez Silva Ribeiro², Talita Oliveira do Vale Lacerda².

\section{RESUMO}

Objetivo: Determinar a prevalência de pinguécula e pterígio em uma população quilombola da Amazônia Brasileira. Metodologia: Estudo transversal, descritivo e analítico realizado com 120 indivíduos de duas comunidades quilombolas (Itancoã e Guajará) da cidade de Acará no estado Pará-Brasil, que foram avaliados por um médico oftalmologista através de exame em lâmpada de fenda e classificados em 1 ou 0 de acordo com a presença e a ausência de pinguécula e pterígio, o sexo e a idade também foram coletados. Os dados foram analisados estatisticamente de forma descritiva e os seguintes testes foram aplicados: binomial, quiquadrado e exato de Fisher, tendo como nível de rejeição da hipótese nula alfa $=0,05$. Resultados: O pterígio teve uma prevalência de 18,3\% (IC95\% = 11,4\% - 25,3\%) na amostra estudada. Enquanto casos de pinguécula foi observado em $7,5 \%$ (IC95\% $=12,2 \%-32,8 \%)$ dos indivíduos examinados. Conclusão: A prevalência de pterígio não alcança $20 \%$ da população quilombola $(p=0,2283)$, e afeta mais homens principalmente a partir dos 40 anos. Enquanto a prevalência de pinguécula é baixa $(p=01144)$, porém os dados encontrados para esta última patologia demandam desenhos de estudos mais acurados para sua melhor investigação.

Palavras Chaves: Oftalmopatia, Pterígio, Pinguécula.

\section{RESUMEN}

Objetivo: Determinar la prevalencia de pinguécula y pterigio en una población quilombola de la Amazonia Brasileña. Métodos: Estudio transversal, descriptivo y analítico realizado con 120 individuos de dos comunidades quilombolas (Itancoã y Guajará) de la ciudad de Acará en el estado Pará-Brasil, que fueron evaluados por un médico oftalmólogo a través de examen en lámpara de hendidura y clasificados en 1 o 0 de acuerdo con la presencia y la ausencia de pinguécula y pterigio, el sexo y la edad también fueron recolectados. Los datos fueron analizados estadísticamente de forma descriptiva y se aplicaron las siguientes pruebas: binomial, qui-cuadrado y exacto de Fisher, teniendo como nivel de rechazo de la hipótesis nula alfa = 0,05. Resultados: El pterigio tuvo una prevalencia del 18,3\% (IC95\% $=11,4 \%-25,3 \%)$ en la muestra estudiada. Mientras que los casos de pinguécula se observó en el 7,5\% (IC95\% = 12,2\% - 32,8\%) de los individuos examinados. Conclusión: La prevalencia de pterigio no alcanza el $20 \%$ de la población quilombola $(p=$

${ }^{1}$ Professora adjunta da faculdade de medicina da Universidade Federal do Pará, Belém- Pará.

${ }^{2}$ Médico da unidade de pronto atendimento de Icoaraci, Belém- Pará. *E-mail: jefisom@yahoo.com.br 
0,2283), y afecta a más hombres principalmente a partir de los 40 años. Mientras que la prevalencia de pinguécula es baja ( $p=01144)$, pero los datos encontrados para esta última patología demandan dibujos de estudios más precisos para su mejor investigación.

Palabras Claves: Oftalmopatía, Pterígio, Pinguécula.

\begin{abstract}
Objective: To determine the prevalence of pinguécula and pterygium in a quilombola population of the Brazilian Amazon. Methods: A cross-sectional, descriptive and analytical study was carried out with 120 individuals from two quilombola communities (Itancoã and Guajará) from the city of Acará in the state of Pará, Brazil, who were evaluated by an ophthalmologist through slit lamp examination and classified in 1 or 0 according to the presence and absence of pinguecula and pterygium, sex and age were also collected. The data were statistically analyzed in a descriptive way and the following tests were applied: binomial, chi-square and Fisher exact, with null hypothesis rejection level alpha $=0.05$. Results: The pterygium had a prevalence of $18.3 \%(95 \% \mathrm{Cl}=11.4 \%-25.3 \%)$ in the sample studied. While pinguecula cases were observed in $7.5 \%$ $(95 \% \mathrm{Cl}=12.2 \%-32.8 \%)$ of the individuals examined. Conclusion: The prevalence of pterygium does not reach $20 \%$ of the quilombola population $(p=0.2283)$, and it affects more men mainly from the 40 years. While the prevalence of pinguécula is low $(p=01144)$, however, the data found for this last pathology require more accurate study designs for its better investigation.
\end{abstract}

Keywords: Ophthalmopathy, Pterygium, Pinguecula.

\title{
INTRODUÇÃO
}

São denominados quilombolas os descendentes dos habitantes dos quilombos, que eram os antigos escravos que formaram comunidades em torno desses núcleos (ITERPA, 2008). Apesar de passado mais de cem anos da abolição da escravidão no Brasil, esses indivíduos continuam em situação de vulnerabilidade, principalmente no que tange ao acesso a serviços de saúde (GOMES et al, 2013). Existem áreas desse tipo espalhadas por todo o país, em algumas regiões a concentração delas é maior, como por exemplo: na Bahia, Maranhão e Pará (ITERPA, 2008). Até outubro de 2013 o Brasil possuía 2408 comunidades quilombolas certificadas pela governo, sem dados precisos sobre a quantidade de indivíduos em cada uma delas (IPEA, 2015). Estudos epidemiológicos abrangendo a população quilombola no estado do Pará são restritos em literatura médica, porém, sabe-se que boa parte dos indivíduos dessas comunidades trabalham em lavouras ou com pescaria (BEZERRA et al, 2013), logo supõe-se que esses sujeitos passam longos períodos expostos a luz solar, poeira e outros resíduos comuns de áreas rurais.

A exposição a essas intempéries podem estar relacionados ao surgimento de diversas afecções, a exemplo disso temos pterígio e pinguécula (YAM e KWOK, 2014) Tanto o pterígio quanto a pinguécula são resultantes de proliferação fibrovascular a partir da conjuntiva, cuja etiologia ainda não é bem conhecida, esse crescimento tecidual no caso do pterígio pode formar uma camada hialina-opaca sobre a córnea podendo levar a redução da acuidade visual, já a pinguécula é um excesso tecidual amarelado que também avança sobre a córnea ao longo do tempo. Ambos podem gerar dor, olho seco e hiperemia. A princípio podem ser realizados tratamentos sintomáticos, porém em casos avançados pode- se indicar a exérese tecidual tanto para melhoria da acuidade visual quanto para melhora estética. (YAM e KWOK,2014; OGUZ, et al.,2001; DUSHKU e REID,1994). Os fatores hereditários ou ambientais como viver em regiões tropicais, sob clima seco e quente tem sido elencado como fatores de riscos para o desenvolvimento dessas oftalmopatias, além da já mencionada relação com a exposição a raios ultraviolentas. Estudos que consideraram a distribuição do pterígio pelo mundo mostraram relação direta entre a taxa de prevalência e a proximidade da localização com a linha do equador (CAMERON, 1962; VOJNIKOVI at al, 2007; THRELFALL E ENGLISH,1999). 
Trabalhos conduzidos no Brasil não apontaram diferenças percentuais entre gêneros (GARRIDO, et al 1996). Porém, tais análises são em maioria feitas com amostras de pacientes que procuram serviço especializado com queixas geradas por essas oftalmopatias ou que realizaram cirurgia para remoção das mesmas(GARRIDO NETO, 1996). A relativa escassez de trabalhos sobre a prevalência de pterígio e pinguécula no Brasil soma-se a dados inexistentes na literatura médica atual sobre a saúde ocular de quilombolas, especialmente no estado do Pará, sendo o principal objetivo deste estudo determinar a prevalência das afecções mencionadas nessas comunidades.

\section{MÉTODOS}

\section{Desenho do Estudo}

Este estudo é transversal, descritivo e analítico, composto por indivíduos pertencentes a comunidades quilombolas de Itancoã e Guajará, localizadas no município de Acará a uma latitude: 01 \$57'39" sul e longitude 48ำ11'48" oeste, estando a uma altitude de 25 metros do nível do mar. O município possui uma população estimada em 54.642 mil habitantes distribuídos em 4.343,805 km² de extensão territorial, fazendo parte do estado do Pará-Brasil, região que faz parte da Amazônia Brasileira. Foram examinados 120 pacientes, com idade variando de 2 a 73 anos de idade, de ambos os sexos.

Nos seis meses que antecederam o estudo, foram realizadas reuniões com os presidentes das comunidades quilombolas, com objetivo de divulgar o atendimento oftalmológico e convidar aqueles indivíduos interessados em participar desta pesquisa. Após estabelecer os dias de atendimento, os quilombolas deslocaram-se de barco a partir de suas comunidades, aos finais de semana, para realizar o exame oftalmológico no Hospital Universitário Bettina Ferro de Souza, localizado na capital paraense.

Os participantes desta pesquisa foram inicialmente esclarecidos sobre os objetivos da avaliação médica e os procedimentos adotados para a realização da mesma. Em seguida Ihes foi requerida permissão escrita para a realização do exame e a utilização dos respectivos resultados para a composição de material científico, bem como foi solicitado a assinatura do termo de consentimento livre esclarecido (TCLE) sobre os procedimentos do estudo e a utilização dos dados coletados, segundo o preconizado pelo Conselho Nacional de Ética em Pesquisa (CONEP) e Comitê de Ética em Pesquisa (CEP) do Núcleo de Medicina Tropical (NMT) da Universidade Federal do Pará UFPA, sendo este último órgão o responsável pela aprovação desta pesquisa, que foi registrada sob código de nํ028/2008-CEP/NMT.

\section{Coleta de Dados}

Os indivíduos que aceitaram participar do estudo foram submetidos a exame oftalmológico, realizado por um médico oftalmologista autor deste estudo. Tal exame foi realizado utilizando lâmpada de fenda Vision Modelo tipo Haag Streiss. Cada indivíduo foi classificado de acordo com a presença e a ausência (pontuado em 1 ou 0 respectivamente) de pinguécula e pterígio, o sexo e a idade também foram coletados.

\section{Análise Estatística}

Os dados coletados receberam análise estatísticas descritivas e inferencial que foi aplicada de duas formas: testes de hipóteses e testes de estimativa de parâmetros. Os testes de hipótese foram aplicados para comparar as prevalências entre os sexos e para avaliar a tendência das afecções na população geral. Foram aplicados os testes: binomial, qui-quadrado e exato de Fisher, efetuados conforme indica Kirkwood (1998) e tendo como nível de rejeição da hipótese nula alfa $=0,05$. A estimativa de parâmetros foi aplicada para estabelecer o intervalo de confiança de $95 \%$ da prevalência dos achados patológicos, conforme prescrito em Ayres (2007). Os procedimentos computacionais foram realizados com o programa Graphpad prism 7.01. 


\section{RESULTADOS}

Dos participantes, $47,5 \%(\mathrm{~N}=57)$ eram do sexo masculino e $52,5 \%(\mathrm{~N}=63)$ do sexo feminino. Quanto à idade, $33,3 \%$ tinham entre 2 e 20 anos, $26,7 \%$ entre $20 \mid-40$ anos, $29,2 \%$ entre $40 \mid-60$ anos e $10,8 \%$ mais que 60 anos. (Tabela 1).

Tabela 1. Distribuição amostral por faixa etária conforme o sexo $(n=120)$.

\begin{tabular}{c|c|c|c|c|c|c}
\hline & \multicolumn{2}{c}{ Masculino } & \multicolumn{2}{c}{ Feminino } & \multicolumn{2}{c}{ Geral } \\
\hline Faixa etária & $\mathbf{N}$ & $\%$ & $\mathbf{N}$ & $\%$ & $\mathbf{N}$ & $\%$ \\
\hline $\mathbf{2}-\mathbf{2 0}$ anos & 24 & 42,1 & 16 & 25,4 & 40 & 33,3 \\
\hline $\mathbf{2 0}-\mathbf{4 0}$ anos & 11 & 19,3 & 21 & 33,3 & 32 & 26,7 \\
\hline $\mathbf{4 0}-\mathbf{6 0}$ anos & 17 & 29,8 & 18 & 28,6 & 35 & 29,2 \\
\hline $\begin{array}{c}\text { Mais que 60 } \\
\text { anos }\end{array}$ & 5 & 8,8 & 8 & 12,7 & 13 & 10,8 \\
\hline TOTAL & 57 & 47,5 & 63 & 52,5 & 120 & 100 \\
\hline
\end{tabular}

Fonte: Dados da Pesquisa, 2016

Entre as patologias em investigação, o pterígio teve a maior prevalência $(18,3 \%)$ (Tabela 2) (IC95\%: $11,4 ; 25,3 \%)$. Observou-se 6 casos de pterígio entre $20 \mid-40$ anos, 7 entre $40 \mid-60$ anos e 9 casos em indivíduos com mais de 60 anos de idade. Houve uma correlação significativa entre a prevalência de pterígio e aumento da idade $(P<0,001)$ (Tabela 3).

Tabela 2. Tabela 2. Prevalência das afecções estudadas $(n=120)$.

\begin{tabular}{c|c|c|c|c|c|c|c}
\hline & \multicolumn{2}{c}{ Masculino } & \multicolumn{2}{c}{ Feminino } & \multicolumn{2}{c}{ Geral } & p- Valor \\
\hline Oftalmopatia & N & $\%$ & N & $\%$ & N & $\%$ & \\
\hline Pterígio & 13 & 28,8 & 9 & 14,3 & 2 & 18,3 & 0,2283 \\
\hline Pinguecula & 2 & 3,5 & 7 & 11,1 & 9 & 7,5 & 0,1144 \\
\hline
\end{tabular}

Fonte: Dados da Pesquisa, 2016

Tabela 3. Distribuição de prevalência de Pterígio por faixa etária $(n=120)$.

\begin{tabular}{c|c|c|c|c}
\hline & \multicolumn{2}{c}{ Caso com pterígio } & \multicolumn{2}{c}{ Caso sem pterigio } \\
\hline Faixa etária & $\mathbf{N}$ & $\%$ & $\mathbf{N}$ & $\%$ \\
\hline $\mathbf{2}-\mathbf{2 0}$ anos & 0 & 0 & 40 & 33,3 \\
\hline $\mathbf{2 0}-\mathbf{4 0}$ anos & 6 & 5 & 26 & 21,7 \\
\hline $\mathbf{4 0}-\mathbf{6 0}$ anos & 7 & 5,8 & 28 & 23,4 \\
\hline Mais que 60 anos & 9 & 7,5 & 4 & 3,3 \\
\hline
\end{tabular}

Fonte: Dados da Pesquisa, 2016

p-valor $<0,0001$ 
A pinguécula ocorreu em 7,5\% das pessoas avaliadas (IC 95\%:12,2\%;32,8\%) (Tabela 2). Estes casos situaram-se entre as faixas de 20 e 60 anos de idade. Como ocorreu com o pterígio houve uma correlação significativa entre a prevalência de pinguécula e aumento da idade $(p<0,0432)$ (Tabela 4).

Tabela 4. Distribuição de prevalência de pinguécula por faixa etária $(n=120)$

\begin{tabular}{c|c|c|c|c}
\hline & \multicolumn{2}{c}{ Caso com pinguécula } & \multicolumn{2}{c}{ Caso sem pinguécula } \\
\hline Faixa etária & $\mathbf{N}$ & $\%$ & $\mathbf{N}$ & $\%$ \\
\hline $\mathbf{2}-\mathbf{2 0}$ anos & 0 & 0 & 40 & 33,3 \\
\hline $\mathbf{2 0}-\mathbf{4 0}$ anos & 6 & 5 & 26 & 21,7 \\
\hline $\mathbf{4 0}-\mathbf{6 0}$ anos & 7 & 5,8 & 28 & 23,4 \\
\hline Mais que 60 anos & 9 & 7,5 & 4 & 3,3 \\
\hline
\end{tabular}

Fonte: Dados da Pesquisa, 2016

$(p<0,0432)$

\section{DISCUSSÃO}

A prevalência de pterígio pode variar bastante de uma localidade para outra, indo desde $0,7 \%$ como a verificada na Dinamarca até 30,8\% encontrada no Japão (NORM,1973; SHIROMA et al.,2009). Muitos são os fatores que foram associados ao surgimento desta alteração ocular (ANG et al., 2012; NANGIA et al.,2013; FOTOUHI et al.,2009), porém, o que o fator mais claramente está relacionado a uma maior prevalência do pterígio em uma determinada população é a proximidade do local onde vivem esses indivíduos com a linha do equador, uma vez que estas áreas tem maior incidência de radiação solar e este parece ser um importante desencadeador desta oftalmopatia (LI e CUI, 2013; LUTHRA et al,,2001; PAULA et al, 2006), altas altitudes também foram relacionadas a uma prevalência relativamente aumentadas (LU et al.,2009). O presente estudo revelou que $18,3 \%$ da população quilombola investigada tem pterígio, dado que foi semelhante ao encontrado em populações indígenas também da floresta amazônica brasileira (PAULA et al.,2006).

Apesar de ser mais comum do que o pterígio em diversos grupos estudados, tendo prevalência que passam de 50\% como foi visto em populações na Austrália (McCarty, 2000) a pingécula teve prevalência relativamente baixa entre os indivíduos deste trabalho, apresentando taxa de $7,5 \%$. Diferindo do que foi encontrado em populações onde foi realizado estudos de prevalência das patologias citadas (PAULA et al.,2006; PANCHAPAKESAN et al.,1998), o pterígio foi mais prevalente neste estudo que a pinguécula entre os quilombolas observados neste trabalho em números absolutos, porém essa diferença não foi estatisticamente significante.

Levando em consideração a prevalência por grupo etário das afecções aqui investigadas, observou-se que o número de casos dessas afecções aumenta com a idade, dado que corrobora com o que foi encontrado em outros estudos (NORM,1979; SUN et al., 2013). O que pode ser explicado também pelo acúmulo de radiação UVB (radiação ultravioleta $\mathrm{B}$ ) ao longo da vida. A análise da prevalência de pterígio por sexo revelou que os homens quilombolas são mais acometidos por essa afeção do que as mulheres, apesar de o Southern Harbin Eye Study (LI e CUI, 2013) e o Handan Eye Study (SUN et al., 2013) terem encontrado a mesma relação, existem trabalhos que apontam uma maior prevalência entre as mulheres, enquanto outros estudos descartam diferenças de prevalências entre sexos. $O$ fato das atividades comuns de áreas rurais (principal ocupação dos quilombolas amazônicos) como pesca e lavoura serem mais exercida por homens (ITERPA, 2008), pode levar estes a terem uma maior exposição solar que na maioria das vezes é feita sem foto proteção, isso explica essa diferença de prevalência entre tais grupos.

Já em relação à pinguécula uma maior prevalência entre as mulheres deste estudo difere do que foi encontrado por Viso et al, 2011 na população em geral da Espanha, que observou que mais de 56\% da

REAS/EJCH | Vol.11 (3) | e144 | DOI: https://doi.org/10.25248/reas.e144.2019 Página 5 de 7 
população afetada por esta afecção foi composta por indivíduos do sexo masculino. O número pequeno de casos de pinguécula entre os quilombolas pode sugerir um viés de amostra já que os indivíduos foram recrutados por demanda espontânea, ou seja, nem todos os indivíduos das comunidades escolhidas foram examinados, uma vez que está oftalmopatia gera menos transtornos visuais, tem crescimento lento e as vezes que complica com pingueculite ( inflamação da pingécula) facilmente pode ser revestida com corticoides tópicos, já o pterígio tem crescimento mais rápido, gera um desconforto estético, além de diminuir a acuidade visual em alguns casos(OGUZ, et al.,2001). Por essas razões pode ter havido um menor recrutamento de pacientes acometidos pela primeira oftalmopatia que pela segunda. Os pacientes que foram diagnosticados com alguma alteração oftalmológica, tanto aquelas que eram objeto de estudo desta pesquisa quanto qualquer outra, foram encaminhados para o serviço de oftalmologia do Hospital Universitário Bettina Ferro de Souza da Universidade Federal do Pará para acompanhamento e tratamento especializado.

\section{CONCLUSÃO}

Concluímos que a prevalência de pterígio não alcança $20 \%$ da população quilombola, e afeta mais homens principalmente a partir dos 40 anos. Enquanto a prevalência de pinguécula é baixa, porém os dados encontrados para esta última patologia demandam desenhos de estudos mais acurados para sua melhor investigação.

\section{REFERÊNCIAS}

1. ANG M; LI X; WONG W et al. Prevalence of and racial differences in pterygium: a multiethnic population study in Asians. Ophthalmology. 2012; 119: 1509-1515.

2. BEZERRA VM, ANDRADE ACS, CÉSAR CC et al. Comunidades quilombolas de Vitória da Conquista, Bahia, Brasil: hipertensão arterial e fatores associados. Cad Saúde Pública. 2013;29(9):1889-902.

3. CAMERON ME. Geographic distribution of pterygium. Trans Ophthalmol Soc Aust. 1962;22:67-81.

4. DUSHKU N., REID T.W. Immunohistochemical evidence that human pterygia originate from an invasion of vimentin-expressing altered limbal epithelial basal cells Curr Eye Res, 13 (1994), pp. 473-481

5. FOTOUHI A; HASHEMI H; KHABAZKHOOB $M$ et al. Prevalence and risk factors of pterygium and pinguecula: the Tehran Eye Study. Eye (Lond). 2009; 23: 1125-1129.

6. GARRIDO N T; GARRIDO C; CARVALHO RC et al. Estudo da frequência de pterígio em Hospitais de Salvador e Manaus. Rev Bras Ofaltamol. 1996;55(9):683-6.

7. GOMES KDO, REIS EA, GUIMARÃES MDC et al. Utilização de serviços de saúde por população quilombola do Sudoeste da Bahia, Brasil. Cad Saude Publica 2013; 29(9):1829-1842.

8. ITERPA- Instituto De Terras Do Pará. Territórios Quilombolas, 2009. Disponível em: http://www.iterpa.pa.gov.br/sites/default/files/caderno_territorios_quilombola.pdf. Acesso em 13 de agosto de 2018.

9. IPEA - Instituto de Pesquisa Econômica Aplicada. Educação escolar quilombola no Censo da Educação Básica. IPEA, Rio de Janeiro, abr. 2015.

10. LI Z; CUI H. Prevalence and associated factors for pterygium in a rural adult population (the Southern Harbin Eye Study). Cornea. 2013; 32: 806-809.

11. LU J; WANG Z; LU P. et al. Pterygium in an aged Mongolian population: a population-based study in China. Eye 2009; 23: 421-427.

12. LUTHRA R; NEMESURE BB; WU SY et al. Frequency and risk factors for pterygium in the Barbados Eye Study. Arch Ophthalmol. 2001; 119: 1827-1832. 
13. MA K; XU L; JIE Y et al Prevalence of and factors associated with pterygium in adult Chinese: the Beijing Eye Study. Cornea. 2007; 26: 1184-1186.

14. MCCARTY CA; FU CL; TAYLOR HR. Epidemiology of pterygium in Victoria, Australia. Br J Ophthalmol. 2000; 84: 289-292.

15. NANGIA V; JONAS JB; NAIR D et al. Prevalence and associated factors for pterygium in rural agrarian central India. The central India eye and medical study. PLoS One. 2013; 8: e82439.

16. NORM MS. Prevalence of pinguecula in Greenland and in Copenhagen, and its relation to pterygium and spheroid degeneration. Acta Ophthalmol 1979;57: 96-105.

17. OGUZ H; KARADEDE S; BITIREN M et al. Tear functions in patients with pinguecula. Acta Ophthalmol Scand. $2001 ; 79(3): 262-265$.

18. PANCHAPAKESAN J; HOURIHAN F; MITCHELL P. Prevalence of pterygium and pinguecula: The Blue Mountains Eye Study. Aust NZ J Ophthalmol 1998;26: 2-5.

19. PAULA JS; THORN F; CRUZ AA. Prevalence of pterygium and cataract in indigenous populations of the Brazilian Amazon rain forest. Eye 2006; 20: 533-536.

20. SUN LP; LV W; LIANG YB et al. The prevalence of and risk factors associated with pterygium in a rural adult Chinese population: the Handan Eye Study. Ophthalmic Epidemiol. 2013; 20: 148-154.

21. SHIROMA H; HIGA A; SAWAGUCHI S et al. Prevalence and risk factors of pterygium in a South-western island of Japan: The Kumejima Study. Am J Ophthalmol 2009; 148: 766-771.

22. -THRELFALL TJ, ENGLISH DR. Sun exposure and pterygium of the eye: a dose-response curve. Am J Ophthalmol 1999; 128: 280

23. VISO E, GUDE F, RODRÍGUEZ-ARES MT. Prevalence of Pinguecula and Pterygium In A General Population In Spain. Eye (Lond) 2011;25(3):350- 357.

24. VOJNIKOVI B; NJIRI S; COKLO M et al. Sunlight and incidence of pterygium on Croatian Island Rab epidemiological study. Coll Antropol 2007; 31(Suppl 1): 61-62.

25. YAM J.C., KWOK A.K. Ultraviolet light and ocular diseases. Int Ophthalmol. 2014;34:383-400. 